\title{
Abordagem posterior para transferência nervosa do nervo acessório para o nervo supraescapular
}

\author{
Ricardo de Amoreira Gepp ${ }^{1}$, José Mauro Cardoso Couto², Marcio Mendonça \\ de Cardoso ${ }^{1}$, Eidmar Augusto Neri², Regis Tavares da Silva ${ }^{2}$
}

Rede Sarah de Hospitais de Reabilitação.

\section{RESUMO}

Objetivo: Os autores realizaram uma avaliação comparativa entre a abordagem anterior e posterior do nervo acessório para transferência nervosa para o nervo supraescapular. Método: Foram estudados pacientes submetidos à neurotização do nervo supraescapular utilizando como doador o nervo acessório, no período de janeiro de 2007 a janeiro de 2010. Os registros cirúrgicos foram avaliados para analisar as vantagens e desvantagens das duas formas de abordagem desses nervos na cirurgia. Foram avaliados os marcos anatômicos utilizados para a localização dos nervos durante a cirurgia e as dificuldades ocorridas nas duas formas de abordagem. Resultados: Foram estudados ao todo 29 pacientes, sendo que 22 deles foram abordados pela via anterior e sete por via posterior. A localização do nervo supraclavicular foi considerada mais fácil pela via posterior, enquanto a localização do nervo acessório foi feita mais rapidamente pela via anterior. Em nenhuma das cirurgias foi necessária a interposição de enxerto nervoso. Não ocorreram complicações cirúrgicas nas duas técnicas. Conclusão: $A$ análise comparativa das duas abordagens cirúrgicas demonstra que a anatomia desses dois nervos é importante fator na dificuldade técnica apresentada na cirurgia. Novos estudos devem analisar se há uma melhora dos resultados funcionais com a abordagem pela via posterior.

\section{PALAVRAS-CHAVE}

Transferência de nervo, nervo acessório, plexo braquial.

\section{ABSTRACT}

Posterior approach to accessory nerve transfer to supraescapular nerve

Objective: The authors conducted a comparative evaluation between the previous approach and later transfer accessory nerve to nerve nervous supraescapular. Method: Patients undergoing the nerve supraescapular transfer to the accessory nerve, in the period January 2007 to January 2010 were studied. Surgical records were evaluated to analyze the advantages and disadvantages of the two ways to approach these nerves in surgery. The anatomical landmarks used for localization of nerves during surgery were evaluated and the difficulties that occurred in the two forms of approaches. Results: Were studied in all 29 patients, with 22 of these were addressed through anterior and 7 through posterior. The location of supraclavicular nerve was considered easier through posterior approach, while the localization of accessory nerve was made more quickly through anterior. In none of the surgeries were required the interposition graft nervous. Non-surgical complications occurred in two techniques. Conclusion: A comparative analysis of two forms of surgical approach demonstrates that knowledge of the anatomy of these two nerves is important factor in technical difficulty presented in surgery. New long-term studies should examine whether there is an improvement of the functional results with the approach through posterior.

\section{KEYWORDS}

Nerve transfer, accessory nerve, brachial plexus.

1. Serviço de Neurocirurgia Rede Sarah de Hospitais de Reabilitação, Brasília, DF, Brasil.

2. Sorocaba, SP, Brasil. 


\section{Introdução}

As lesões da porção superior do plexo braquial (C5 e C6) ocasionam déficit de função do ombro e de flexão do cotovelo. ${ }^{3}$ Esses movimentos são importantes para o funcionamento do membro superior, servindo o ombro como apoio e centro para a flexão do antebraço sobre o braço, permitindo, assim, uma ação efetiva da mão do paciente. A transferência do nervo acessório para o nervo supraescapular é um dos procedimentos cirúrgicos mais utilizados para restabelecer a função de abdução do ombro executado pelo músculo supraespinhoso. ${ }^{2}$ Classicamente, a abordagem desses nervos é realizada cirurgicamente pela via anterior, na região supraclavicular. ${ }^{3,4}$ Mais recentemente, descrições têm sido feitas a respeito da abordagem posterior desses nervos, principalmente quando associada à transferência nervosa de ramos do nervo radial para o nervo axilar. ${ }^{1}$

Os autores realizaram uma avaliação comparativa entre as técnicas cirúrgicas de abordagem anterior e posterior do nervo acessório para transferência nervosa para o nervo supraescapular, em pacientes portadores de lesão do plexo braquial, descrevendo os detalhes técnicos e avaliando as vantagens e desvantagens de cada método cirúrgico.

\section{Método}

Foram estudados pacientes vítimas de lesão completa ou parcial do plexo braquial, que foram submetidos à neurotização do nervo supraescapular utilizando como doador o nervo acessório. A avaliação retrospectiva dos prontuários compreendeu os pacientes operados no período de janeiro de 2007 a janeiro de 2010. Todos os pacientes foram submetidos à avaliação clínica por examinador independente e a pelo menos um exame neurofisiológico - os casos selecionados tinham que apresentar exame clínico e eletroneuromiografia demonstrando que não houve lesão associada do nervo acessório. Todos os casos analisados foram submetidos a estudo de imagem pré-operatória, por meio de ressonância magnética nuclear ou mielotomografia da região cervical. Os registros cirúrgicos foram avaliados para analisar as vantagens e desvantagens das duas formas de abordagem desses nervos na cirurgia. Foram estudados os marcos anatômicos utilizados para a localização dos nervos durante a cirurgia e as dificuldades ocorridas nas duas formas de abordagens. A seguir, descrevemos as técnicas cirúrgicas utilizadas.

\section{Técnica cirúrgica}

Via anterior: Paciente posicionado em decúbito dorsal, com a cabeça vertida para o lado contralateral a abordagem cirúrgica, é feito uma incisão transversal na porção lateral cervical externamente ao músculo esternoclidomastoídeo. Após a abertura do músculo platisma, é feita a dissecção do plexo braquial com identificação do nervo supraescapular após a sua saída do tronco superior. O nervo acessório é localizado próximo à porção superior do plexo cervical e apresenta como marco anatômico superficial a emergência dos nervos auricular maior e occipital menor. Após a localização do nervo acessório este é dissecado distalmente para que o nervo alcance sem tensão na sutura o nervo supraescapular. A anastomose é feita com microcirurgia utilizando fio mononylon 9-0.

Via posterior: $\mathrm{O}$ paciente é posicionado em decúbito ventral. São feitas as marcações de pontos de referência na pele para a localização dos nervos (Figura 1). Para identificação do nervo acessório, traça-se uma linha ao longo da borda superior da escápula, entre o processo espinhoso e o acrômio, e marca-se o ponto do nervo cerca de $40 \%$ da distância partindo-se da linha média. O músculo trapézio é aberto na direção das suas fibras e utiliza-se o estimulador de nervo que auxilia na sua posição. Para a localização do nervo supraescapular, o ângulo superior da escápula e o acrômio são marcados; no ponto médio entre estas duas referências encontra-se o nervo supraescapular. Após a incisão, o músculo trapézio é aberto para em seguida realizar a palpação da borda superior da escápula e localizar a incisura escapular onde se encontra o nervo. Após a abertura do ligamento, o nervo supraescapular está liberado para receber o nervo acessório que é rebatido em sua direção. A sutura é feita com fio 9-0 e utilizando o microscópio cirúrgico (Figura 2).

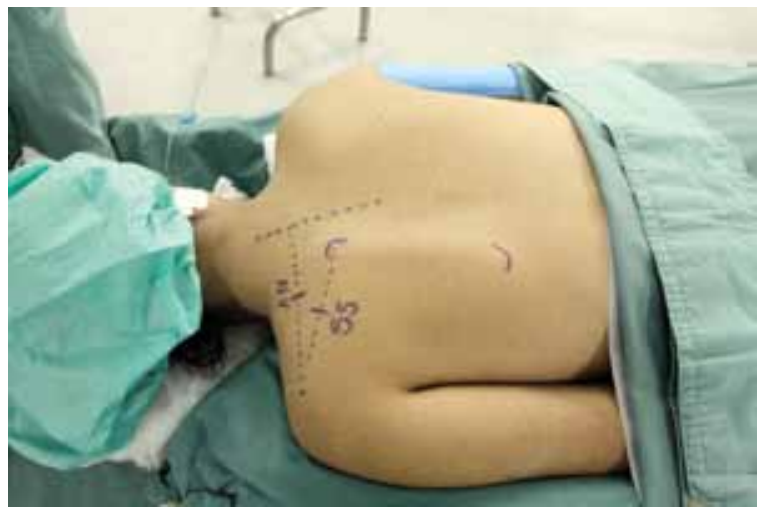

Figura 1 - Paciente em decúbito ventral para a realização da abordagem posterior. A linha superior traçada entre a linha média da coluna e o acrômio mostra a posição do nervo acessório (AN). A linha inferior representa a escápula e o nervo supraescapular (SS). 

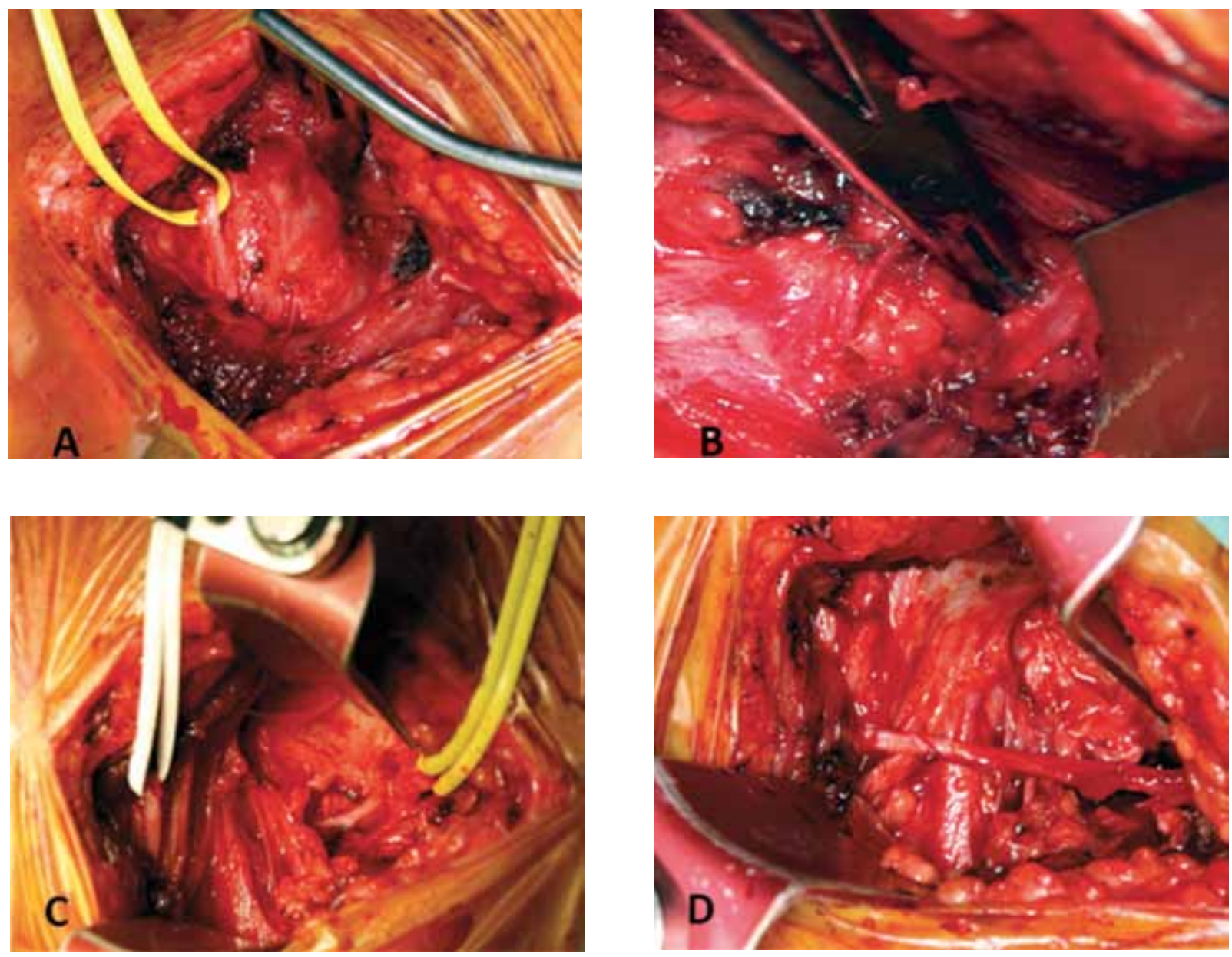

Figura 2 - (A) Exposição do nervo acessório. (B) Liberação do nervo supraescapular na incisura da escápula. (C) Nervos acessório e supraescapular expostos. (D) Anastomose entre os dois nervos sem interposição de enxerto.

\section{Resultados}

Foram estudados por meio de revisão de prontuários ao todo 29 pacientes - 22 deles foram abordados pela via anterior e 7 por via posterior. Os pacientes do sexo masculino eram 27 do total. A lesão completa do plexo braquial ocorreu em 16 casos, e nos 13 restantes a lesão foi parcial, com acometimento superior do plexo. Nos casos em que houve a lesão superior, sete pacientes apresentaram alterações em C5, C6 e C7, enquanto em seis casos a lesão era apenas de C5 e C6.

$\mathrm{Na}$ análise dos relatos cirúrgicos, a localização do nervo supraescapular foi considerada mais fácil pela via posterior por causa dos pontos anatômicos de referência na escápula. Na via anterior, o nervo supraescapular necessitou de maior dissecção e, em lesões graves do plexo, a sua localização foi mais demorada, principalmente quando havia lesões associadas do tronco superior.
A localização do nervo acessório foi feita mais rapidamente pela via anterior em virtude dos marcos anatômicos do plexo cervical superficial. A incisão cirúrgica variou entre a transversal acima da clavícula e longitudinal seguindo a borda lateral do músculo esternoclidomastoídeo. A presença dos marcos anatômicos baseados nos nervos superficiais do plexo cervical facilitou a localização do nervo acessório na superfície do músculo levantador da escápula. O nervo acessório apresentou uma maior dificuldade de ser localizado pela via posterior, mas a utilização do estimulador de nervo foi muito útil nessa localização após a abertura do músculo trapézio.

Em todos os procedimentos cirúrgicos, foram possíveis a localização dos nervos e a realização da anastomose direta sem a necessidade de interposição de enxerto de nervo sural. Não ocorreram lesões vasculares, danos a outros nervos ou complicações cirúrgicas nas duas técnicas. Não houve nenhum caso 
de infecção pós-operatória superficial ou profunda nesta série de casos. A dor pós-operatória não foi significativa nas duas formas de abordagem cirúrgica e não se observou restrição na mobilização do ombro ou pescoço pelas intervenções.

\section{Discussão}

Classicamente, o tratamento das lesões do plexo braquial era feito pela abordagem cirúrgica direta ao plexo, com estudo neurofisiológico e reparo direto da lesão. ${ }^{3}$ Mais recentemente, a realização de transferências nervosas modificou a forma de tratamento e tornou-se a forma preferencial de tratamento das lesões de plexo braquial para vários autores. ${ }^{2,5-7}$

As transferências distais apresentam vantagens em relação à cirurgia direta do plexo, pois não necessitam de ampla dissecção na região do plexo braquial e permitem a inervação próxima ao músculo efetor do movimento. Além desses dados, as respostas funcionais têm se demonstrado superiores à abordagem direta ao plexo braquial.

Inicialmente, a via preferencial da transferência nervosa do nervo acessório para o nervo supraescapular é a abordagem cervical na região supraclavicular. Apenas mais recentemente, maior atenção tem sido dada à via posterior, principalmente após o estudo de Colbert e Mackinnon ${ }^{1}$ sobre a dupla transferência posterior. Anteriormente pouco descrita, a via de acesso posterior ao plexo braquial tem sido mais utilizada, principalmente quando o objetivo da cirurgia é o tronco inferior, demonstrando, assim, que o acesso cirúrgico não deve ser padronizado, e sim definido pelo objetivo da cirurgia. ${ }^{6,8}$

A análise comparativa das duas formas de abordagem cirúrgica demonstra que o conhecimento da anatomia desses dois nervos é importante fator para a diminuição da dificuldade técnica apresentada na cirurgia. Apesar de necessitar de reposição do paciente para o decúbito ventral, a via posterior tem sido a opção preferencial, na maioria dos casos devido a três fatores: a fácil localização dos nervos após familiarização com a técnica cirúrgica, a possibilidade de ocorrer uma anastomose direta sem interposição de enxerto nervoso e a transferência nervosa ocorrer mais próximo do músculo efetor do movimento, no caso o músculo supraespinhoso. Novos estudos em longo prazo devem analisar se há uma melhora dos resultados funcionais com a abordagem pela via posterior.

As duas formas de realização da anastomose do nervo acessório com o nervo supraescapular são efetivas. Atualmente, utiliza-se a via posterior como forma de eleição para essa anastomose, mas em situações em que não há necessidade de realização de nenhum procedimento em decúbito ventral utiliza-se a via anterior (Tabela 1). Nos pacientes em que será feita a transferência de ramo do nervo radial para o nervo axilar, utiliza-se preferencialmente a abordagem posterior. A não necessidade da mudança de decúbito do paciente, quando este é submetido à anastomose axilar com o nervo radial, é um fator importante na redução do tempo cirúrgico. Nos casos em que será feita apenas uma anastomose do nervo ulnar para o nervo musculocutâneo, usa-se preferencialmente a via anterior, mas pode ser feita também a via posterior.

\section{Conclusão}

Esta série de casos demonstrou que a realização da transferência do nervo acessório para o nervo supraescapular pela via posterior é efetiva e prática, principalmente quando da necessidade da realização de outras transferências com o paciente em decúbito ventral. As dificuldades técnicas são resultantes do conhecimento anatômico e da experiência com a via de acesso, mas no momento não há dados que demonstrem que o resultado funcional é melhor. Estudos futuros comparando os resultados funcionais entre as duas formas de abordagem são necessários.

\begin{tabular}{lll}
\hline & Tabela 1 - Resumo das propostas de via de acesso em relação ao objetivo da cirurgia de transferência nervosa \\
\hline Lesão do plexo & Cirurgia proposta & Via de acesso acessório-supraescapular \\
Completa & Anastomose frênico-musculocutâneo & Anterior \\
Incompleta & Anastomose radial-axilar & Posterior \\
Incompleta & Anastomose ulnar-musculocutâneo & Anterior ou posterior \\
\hline
\end{tabular}




\section{Referências}

1. Colbert SH, Mackinnon S. Posterior approach for double nerve transfer for restoration of shoulder function in upper brachial plexus palsy. Hand (N Y). 2006;1(2):71-7.

2. Colbert SH, Mackinnon SE. Nerve transfers for brachial plexus reconstruction. Hand Clin. 2008;24(4):341-61.

3. Kline DG. Surgical repair of brachial plexus injury. $J$ Neurosurg. 2004;101(3):361-3.

4. Kline DG. Timing for brachial plexus injury: a personal experience. Neurosurg Clin N Am. 2009;20(1):24-6.

5. Mackinnon SE, Colbert SH. Nerve transfers in the hand and upper extremity surgery. Tech Hand Up Extrem Surg. 2008;12(1):20-33.

6. Mackinnon SE, Novak CB, Myckatyn TM, Tung TH. Results of reinnervation of the biceps and brachialis muscles with a double fascicular transfer for elbow flexion. J Hand Surg Am. 2005;30(5):978-85.

7. Sulaiman OA, Kim DD, Burkett C, Kline DG. Nerve transfer surgery for adult brachial plexus injury: a 10-year experience at Louisiana State University. Neurosurgery. 2009;65(4 Suppl):A55-62.

8. Tender GC, Kline DG. Posterior subscapular approach to the brachial plexus. Neurosurgery. 2005;57(4 Suppl):377-81.

\section{Endereço para correspondência}

Ricardo de Amoreira Gepp

Rua SQSW, 300, bloco M, ap. 204, Sudoeste

70673-014 - Brasília, DF, Brasil

Telefone: (61) 3342-2420

E-mail: ricardogepp@zipmail.com.br; rgepp@sarah.br 